\title{
Truth or dare on mineral inclusions
}

Matteo Alvaro ${ }^{1 *}$, Ross J. AnGeL ${ }^{2}$, Mattia L. MAZZUCCHELLI $^{1}$

${ }^{1}$ Dipartimento di Scienze della Terra e dell'Ambiente, Università di Pavia, Italy

(*correspondence:matteo.alvaro@unipv.it)

${ }^{2}$ Istituto di Geoscienze e Georisorse, CNR, Padova, Italy

Investigations of mineral inclusions still trapped in their host can provide a wealth of information on the architecture and evolution of the Earth's lithosphere through time. Reconstructions of the geological processes governing crustal subduction and uplift rely on correct estimates of the pressures and temperatures (P-T) experienced by rocks over time. Unlike chemical geothermobarometers, elastic geobarometry on mineral inclusions does not rely on chemical equilibrium between minerals, so it has the potential to provide information on over-stepping of reaction boundaries and to identify other examples of non-equilibrium behaviour in rocks. Recent extensive development of elastic geobarometry allows us to reliably determine crystallization conditions for inclusions in their hosts, although some limitations on the methodology still exist.

We illustrate these concepts, advantages and limitations by applying elastic geobarometry to natural rocks from different ultra-high-pressure terrains and to some synthetic host inclusion systems for which the $\mathrm{P}$ and $\mathrm{T}$ conditions of crystallization and entrapment are known. For encapsulation conditions very close to hydrostatic these methods allow the strain state to be determined and, in turn, the stress state experienced by the inclusions upon subduction and exhumation. Conversely, even though quantitative measurements are still not possible, these results at least allow us to infer the occurrence of local deviations from hydrostatic stress at the time of entrapment and/or post entrapment for some inclusion suites.

This work was financially supported by the European Research Council (ERC) under the European Union's Horizon 2020 research and innovation program GRANT agreements 714936 to M. Alvaro 